\title{
Target discovery focused approaches to overcome bottlenecks in the exploitation of antimycobacterial natural products
}

\author{
Rafael Baptista1, Sumana Bhowmick1, Robert J Nash², Les Baillie ${ }^{3}$ \& Luis AJ Mur ${ }^{\star 1} 1$ \\ ${ }^{1}$ Institute of Biological, Environmental \& Rural Sciences, Aberystwyth University, Penglais Campus, Aberystwyth, Wales, UK, SY23 \\ 2DA \\ 2Phytoquest Ltd, Plas Gogerddan, Aberystwyth, UK SY23 3EB \\ ${ }^{3}$ School of Pharmacy \& Pharmaceutical Sciences, Cardiff University, Redwood Building, Cardiff, Wales, UK, CF10 3NB \\ * Author for correspondence: lum@aber.ac.uk
}

Tuberculosis is a major global health hazard. The search for new antimycobacterials has focused on such as screening combinational chemistry libraries or designing chemicals to target predefined pockets of essential bacterial proteins. The relative ineffectiveness of these has led to a reappraisal of natural products for new antimycobacterial drug leads. However, progress has been limited, we suggest through a failure in many cases to define the drug target and optimize the hits using this information. We highlight methods of target discovery needed to develop a drug into a candidate for clinical trials. We incorporate these into suggested analysis pipelines which could inform the research strategies to accelerate the development of new drug leads from natural products.

First draft submitted: 1 November 2017; Accepted for publication: 25 January 2018; Published online: 23 March 2018

Keywords: natural products $\bullet$ target discovery $\bullet$ tuberculosis

\section{Natural products: moving back to the forefront of antimycobacterial target discovery}

Tuberculosis (TB) is the world's leading cause of death from infectious disease, causing an estimated 1.4 million deaths in 2015 [1]. This effectively represents a reverse of trends seen during the middle years of the 20th century when antibiotics, developed from the 1940s, appeared to be effective in controlling the disease. However, over the last two decades TB is again a major public health hazard with the appearance of drug-resistant strains of TB, multidrug-resistant TB (MDR-TB), extensively drug-resistant TB (XDR-TB) and more recently strains resistant to all the antitubercular chemotherapies [2]. This situation has arisen for a great extent due to the complex drug therapy regime used for TB which reduces patient compliance and adherence to prescribed medication. Thus, antitubercular chemotherapy comprises at least a 6-month drug regimen involving an initial 2-month phase of four agents (isoniazid, rifampicin, pyrazinamide and ethambutol) followed by an additional 4 months with isoniazid and rifampicin [3,4].

MDR-TB is resistant to both ifampicin and isoniazid, the most effective antituberculous drugs. XDR-TB strains are resistant to at least rifampicin and isoniazid but also fluoroquinolones and to, at least, one of the injectable drugs; capreomycin, kanamycin or amikacin. Treatment of MDR-TB consists of a 2-year therapy with a combination of four to six first- and second-line antitubercular drugs [5]. TB control is therefore contingent on the development of new drugs and in the past decade there have been major efforts made in this area. There is also a need for new drugs that act quickly, giving fewer opportunities for the TB-causing organism to develop resistance. Any new drugs also need to be inexpensive to produce, so that it can be widely adopted in countries outside of the first world. Due to renewed research and development efforts, bedaquiline, a diarylquinolone, became the first anti-TB drug approved by the US FDA in more than 40 years [6]. Additionally, innovative methodologies have allowed the development of more effective therapies, the 'revitalization' of old drugs, re-use of drugs in different contexts, and the reduction of drugs rejected due to their toxicity profile. A vitally important aspect of research leading to new drugs or use of 
existing drugs is a requirement to fully comprehend, at least as far as possible, their mechanisms of action and, this way, prevent problems with toxicity at the early stages of clinical trials.

In the early part of the last century, research into natural products was prominent in the search for new drug leads. Indeed, the attractiveness of natural products is obvious considering they reflect the outcome of evolutionary pressures acting on genomes to produce bioactives to serve many roles including antimicrobial [7]. Streptomycin, isolated from actino-bacterium Streptomyces griseus, is an important example and on first introduction was as a standard treatment against TB. However, with the emergence of drug-resistant strains of TB and its increased prevalence with the onset of human immunodeficiency virus/acquired immune deficiency syndrome (HIV/AIDS), streptomycin has been replaced by other therapies. Streptomycin is also problematic due to its oto- and nephrotoxicity with prolonged therapies [8]. With the advent of combinatorial chemistry and highthroughput screening strategies, drug discovery focusing on natural products became less popular. It was thought that screening vast combinatorial libraries would speed up the development process by eliminating lengthy natural product identification and often complex synthesis programs. As a result of the combinatorial library focus, very few antibiotics, derived or based on natural products, had been approved in the past two decades by the FDA.. However, the poor hit rate though of the combinatorial approach and low diversity of the libraries led to some big Pharma companies abandoning this approach and looking for new and better ways to explore natural products.

The importance of a good antimycobacterial therapy does not reside only on drug potency and safety, but also in some key features that targets should display. Kana et al. defines the 'ideal' target as a macromolecule that acts in the growth, survival and latency of the bacteria, regulates crucial checkpoints of bacterial metabolism, has a low tolerance for mutation and is found at targetable locations within the cell. Additionally, the drug target must not show such similarity with human homologs enough to compromise the drug safety [9]. To meet such requirements many groups favor an approach where drugs are derived to known mycobacterial proteins. Besides, avoiding the possibility of new, unexpected targets being defined, this approach could be flawed since it does not consider the cell environment as essential to the drug's mode of action. Thus, several details are not evaluated; for example, the pharmacokinetics of drug internalization by the mycobacteria, innate resistance mechanisms (e.g., efflux pumps) and the physico-chemical properties of both bacteria and compound. Consequently, potent inhibitors of a specific target can display unsatisfactory values of in vitro minimum inhibitory concentration (MIC) against Mycobacterium species [10]. One example of target-based drug discovery that has recently failed is isocitrate lyase, a key enzyme within the glyoxylate shunt pathway. This enzyme was suggested as a suitable target since it is essential to the growth and virulence of $M$. tuberculosis in both acute and chronic phases of infection [11]. This work was discontinued most likely to the target active site being 'shallow' and lack of hydrophobic patches that could be targets for inhibitors [12]. None of the drugs that are currently in clinical trials have arisen following a target-to-drug approach and instead these have tended to follow drug-to-target strategies based on whole cell-based screenings [13].

It is against this background that natural product discovery based on whole-cell screenings has become a viable strategy to provide druggable molecules. However, this strategy should be combined with innovative '-omics' technologies, chemical biology and genetics which have made possible the discovery of new drug and target leads [14]. This is especially useful in the search for new agents against $M$. tuberculosis where natural products have assumed a prominent role [15]. The potential of natural products as antimycobacterial drug leads lies in their intrinsic cell permeability and structural, functional and stereochemical diversity, that provide unique scaffolds for further drug optimization toward increased potency and selectivity [16,17]. Moreover, new natural products could be a useful tool to unveil the role of several proteins that are transcribed from the $M$. tuberculosis genome but whose functions are unknown [18,19].

These points, notwithstanding the recent failures to take forward natural products or their derived synthetics, needs to be recognized and the underlying causes identified. We suggest that these arise through incomplete workflow methodologies that massively affect further development of an antitubercular drug. A cursory glance at the literature will show many examples of antimycobacterial activity being detected in a natural product but these observations are not further characterized and can be thought of as effectively useless from the perspective of defining a new drug lead. In particular, the unequivocal identification of the biological target coupled with a clear mechanism of action of the bioactive is often not done [20]. This is essential to allow the reduction in the number of microbial 'off-targets' and toxicity to human systems and so leading to the development of potent and safe drugs against $M$. tuberculosis.

This review will focus on antimycobacterial target discovery methodologies and highlight examples of 'best practice' where natural product research has included optimization of target identification and validation steps 


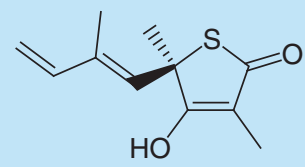

Thiolactomycin

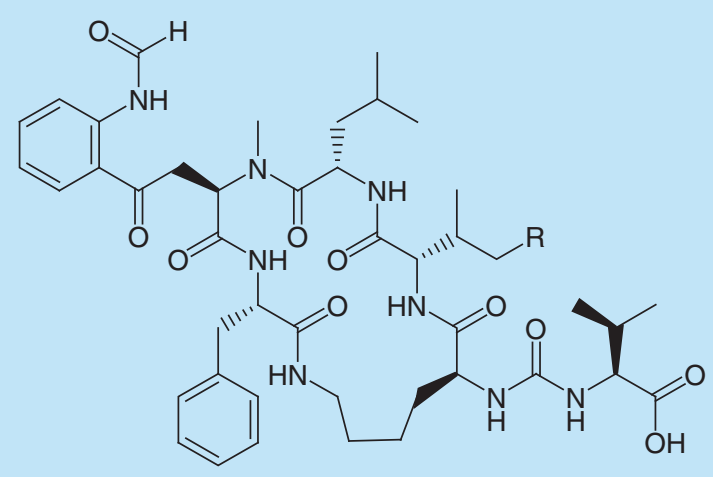

Brunsvicamide $\mathrm{B} \quad \mathrm{R}=\mathrm{H}$

Brunsvicamide $\mathrm{C} \quad \mathrm{R}=\mathrm{CH}_{3}$<smiles>COc1ccc2cc3[n+](cc2c1OC)CCc1cc2c(cc1-3)OCO2</smiles>

Berberine

Figure 1. Chemical structures of thiolactomycin, brunsvicamide $B$ and $C$ and berberine.

(Table 1) [21]. In doing so, it will identify potential pipelines for target discovery and validation which could inform research strategies based on natural products. For readers wishing to consider only the discovery of antimycobacterial natural products, we recommend the following review articles [22-24].

\section{Methodological options for target discovery Biochemical approaches}

The 'classical' approach to target discovery and validation aims to assess biochemical differences arising following treatment with the natural product. Such change could arise from the perturbation of enzymatic activities and these approaches can give valuable insights of the mode of action and target. Putative mycobacterial targets that have been explored using this strategy can be classified according to the pathway they affect. Thus, notable biochemical targets have proven to be in peptidoglycan biosynthesis, arabinogalactan and lipoarabinomanan biosynthesis, mycolic acids biosynthesis, shikimate pathway, pantothenate biosynthesis, biotine pathway, glyoxylate shunt pathway and DNA metabolism [25,26].

Scientists following such biochemical approaches have provided some essential insights to a robust, multifaceted pipeline that can yield some potentially druggable antimycobacterials. Thiolactomycin (TLM) (Figure 1) was isolated from Nocardia sp., and displays an MIC of $25 \mu \mathrm{g} / \mathrm{ml}$ against $M$. tuberculosis [27]. Despite the quite high MIC, TLM's physicochemical properties, its low cytotoxicity in ex vivo macrophage models and high in vivo availability, are features that encouraged its further development [27-29]. Several studies have revealed that both $\beta$-ketoacyl-ACP synthases I/II and acetyl coenzyme-A (CoA) were targets of TLM in E. coli [30]. Based on the incorporation of radiolabeled isotopes into fatty acids and mycolic acid metabolism, it was concluded that TLM reversibly inhibits the synthesis of both fatty acids and mycolic acids with a high selectivity (at $0.75 \mu \mathrm{g} / \mathrm{ml}$ ) for Fas-II $\beta$-ketoacyl-ACP synthase [31]. Kremer et al. overexpressed KasA and KasB, two synthases belonging to the Fas-II family, to validate these as targets of TLM [32]. Several analogs of TLM, either in racemic mixtures or as pure 


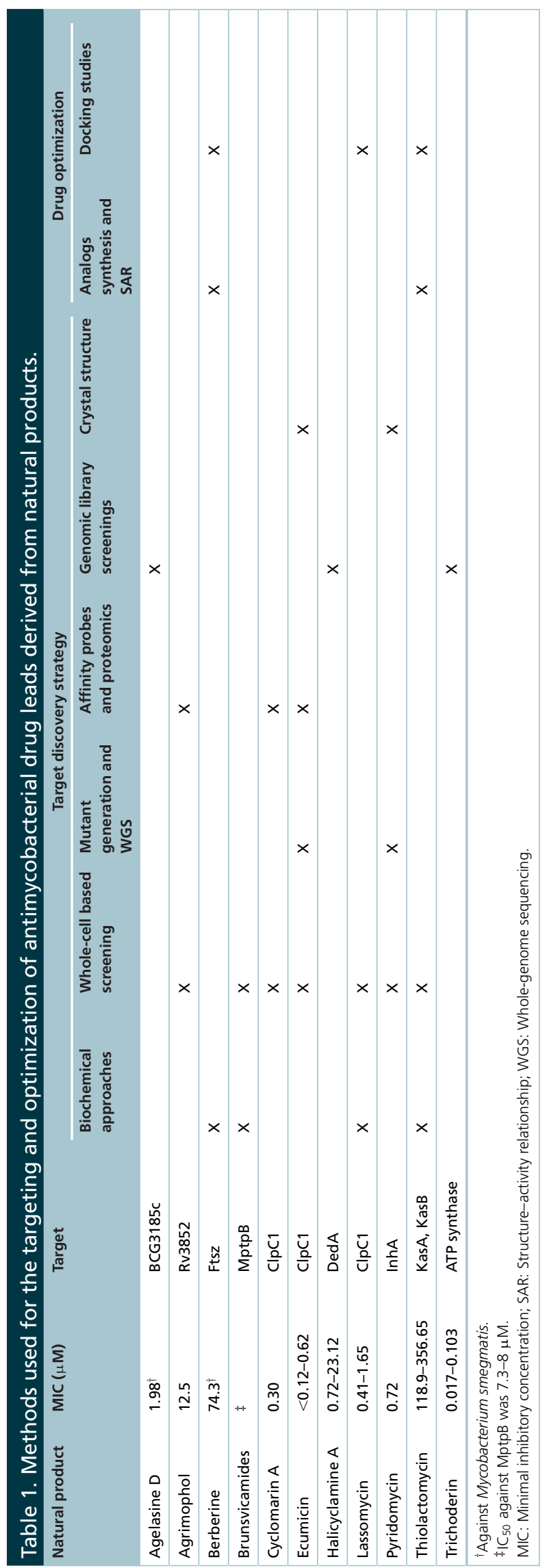


enantiomers, have been synthesized and tested in vitro against $M$. tuberculosis [33]. When considered together, these TLM analogues will contribute to structure-activity relationship (SAR) studies, vital for TLM optimization. Thus, a dataset of 40 TLM analogs, $\beta$-ketoacyl-ACP synthase III ( $\mathrm{mtFabH}$ ) inhibitors were subjected to a 3D-quantitative structure-activity relationship (3D-QSAR) analysis. The derived QSAR model and associated docking studies is informing our understanding of the mechanism of TLM as an mtFabH inhibitor [34].

Brunsvicamides are cyclic hexapeptides that were first isolated from the cyanobacterium Trychonema. These, especially Brunsvicamide B and C (Figure 1), were shown to target protein tyrosine phosphatase B (MptpB) which is an essential virulence factor of mycobacterial species. The activity of these compounds were successfully assessed against a range of protein phosphatases where $\mathrm{MptpB}$ showed an $\mathrm{IC}_{50}$ of $7.3 \mu \mathrm{M}$ [35]. The characterization of MptpB as the target directly led to the identification of novel synthetic inhibitors of this protein using high-throughput screens [36]. These, along with diverse natural variants could provide sufficient chemical diversity to perform SAR studies of MptpB and therefore new treatments for tuberculosis [37].

Another example is berberine (Figure 1), a natural benzylisoquinoline alkaloid obtained from the plant genus Berberis. This was found to be active against $M$. smegmatis and MDR-TB at $25 \mu \mathrm{g} / \mathrm{ml}$ [38]. Studies which included in silico molecular docking analysis revealed the target to be a protein called Ftsz [39]. This protein forms a ring to aid in the formation of the septum between dividing bacterial cells. Additionally proper ordering of the septum is dependent on FtsZ GTPase activity. Berberine was found to inhibit Fstz ring assembly in a dose-dependent manner by a real-time light-scattering assay and GTPase activity using a GTP hydrolysis assay. The binding between Ftsz and berberine were confirmed by isothermal titration calorimetry. The clear identification of this protein as the berberine target led directly to the derivation of berberine analogues aimed at an SAR analysis using 2D- and $3 \mathrm{D}-\mathrm{Q} S A R[40,41]$. The relative success of these analyses will be an important determinant in the optimization of berberine as a potential drug.

\section{Genomic-based approaches}

Some of the more recent approaches used in drug target discovery take advantage of the great advances in genome sequencing capabilities. These encompass a wide variety of approaches including transcriptomic analyses and DNAprotein binding studies based on chromatin immunoprecipitation (ChIP). In this approach, antibodies are used to enrich for particular chromatin components and the relative amount of associated DNA sequences can reveal epigenetic control points [42].

Considering genomic approaches to search for antimycobacterials targets, some are based on screening genomic libraries for clones that could confer tolerance when expressed in heterologous hosts. This methodology was used to identify the targets for halicyclamine $\mathrm{A}$, trichoderin $\mathrm{A}$ and agelasine $\mathrm{D}$, three natural products derived from the marine sponges Haliclona sp. 05A08, Trichoderma sp. and the genus Agelas, respectively (Figure 2) [43-45]. Genomic cosmid libraries were constructed from strains of M. smegmatis and M. bovis BCG which exhibited spontaneous resistance to one of the above compounds and each cosmid was introduced into a susceptible strain. Cosmids with derived alleles that are mutated in the target(s) could increase the MIC required to affect the recipient mycobacterial strain. By following this strategy, DedA was identified as the target of halicyclamine A and BCG3185c as the target for agelasine $\mathrm{D}[46,47]$, but unfortunately, these results were not validated by other approaches. In contrast, cosmid screening suggested that trichoderin A targeted ATP synthase, which was validated by measurements of the ATP content in M. bovis BCG [48]. The first total synthesis of trichoderin A has recently been described and this will be essential in SAR studies [49].

Moving beyond cosmid library screening, whole-genome sequencing (WGS) analyses are assuming a very significant role in target discovery by (for example) identifying distinctive genes present only within Mycobacterium. However, this powerful approach needs to be carefully used as suggested targets can be intractable, nonessential or undrugabble [50]. Thus, we suggest that the genomic information should be used within a combined methodology pipeline complemented by validation techniques based on target discovery on tuberculosis [13]. This pipeline could be as follows. First, the MIC must be confirmed using whole mycobacterial cell based in vitro screens. These will inform what concentrations of natural target to use in suggestive rounds of culturing with the aim of deriving variants which exhibit resistance. Such resistance could arise from target overexpression of deletion, transcriptional or translational disruption or mutation of a key motif. Bacterial DNA from mutants that are resistant to high concentrations of the specific molecule is extracted and sequenced, aiming at confirmation of the mutational site [51]. 


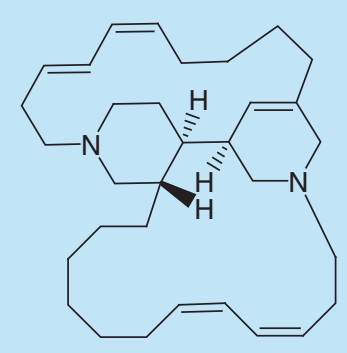

Halicyclamine A

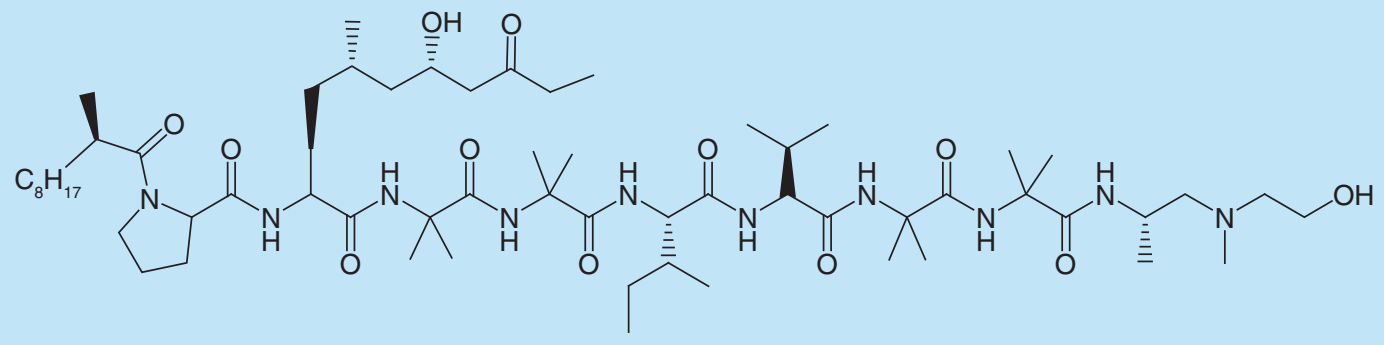

Trichoderin A

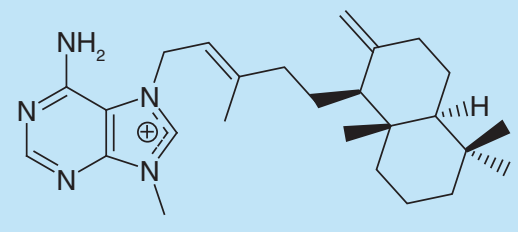

Agelasine D<smiles>CC/C(C)=C(\OC(=O)[C@@H](C)[C@@H](O)[C@@H](Cc1cccnc1)NC(=O)[C@H](NC(=O)c1ncccc1O)[C@@H](C)OC(=O)c1ncccc1O)C(=O)O</smiles>

Pyridomycin<smiles>CCC(C)[C@H](NC(=O)[C@H](CC(N)=O)NC(=O)[C@H](CCCNC(=N)N)NC(=O)[C@H](CCCNC(=N)N)NC(=O)CCC(=O)[C@H](NC(=O)[C@H](CC(C)C)NC(=O)[C@H](CCC(N)=O)NC(=O)[C@@H]1CC(=O)NC(=O)N[C@H](CC(C)C)C(=O)N[C@@H](CC(C)C)C(=O)N[C@@H](CCCNC(=N)N)C(=O)N[C@@H](CCCNC(=N)N)C(=O)N1)C(C)C)C(=O)OC</smiles>

Lassomycin

Figure 2. Chemical structures of halicyclamine A, trichoderin A, agelasine D, pyridomycin and lassomycin.

This seemingly straight-forward strategy may be confounded by the up-regulation of bacterial defence mechanisms such as efflux pumps. Indeed, increased activity of efflux pumps may be a bacterial response to the xenometabolite, rather than direct genetic alteration of operons encoding mutations in the efflux pumps. In this context, it is relevant that antibiotic resistance clinical isolates do not show any mutations in the genes and regulators of bacterial efflux systems. As a result the efflux pumps can provide 'false-positives' in other words, strains where there have been no mutations events linked to the bioactive target. Thus, Ioerger et al. suggested the inclusion of an efflux-pump inhibitor then screening for mycobacterial resistance to any bioactive [51]. Suitable efflux-pump inhibitors could be natural products such as reserpine or verapamil [52]. 
The discovery of the recent natural product lead against TB, pyridomycin (Figure 2), is an example of a complete and validated target discovery based research, which included genomic analyses. Pyridomycin, was isolated from Dactylosporangium fulvum and Streptomyces pyridomyceticus, and exhibited a MIC of $0.39 \mu \mathrm{g} / \mathrm{ml}$ against $M$. tuberculosis. Hartkoorn and his colleagues identified InhA, an NADH-dependent enoyl-Acyl-Carrier-Protein as the principal target of pyridomycin following WGS of a resistant mutant colony of M. tuberculosis. Although InhA is also the target of isoniazid, pyridomycin behaves as a competitive inhibitor of this protein as shown by its activity against isoniazid-resistant clinical isolates. To validate the target, wild-type $i n h A$ and the mutant form was overexpressed in $M$. tuberculosis $\mathrm{H} 37 \mathrm{Rv}$ and this allowed the steady-state kinetics and susceptibility to isoniazid and pyridomycin to be accurately measured [53].

Insights on lassomycin (Figure 2) mode of action were also made using a WGS approach. Lassomycin was isolated from Lentzea kentuckyensis spp. IO0009804 and exhibits MIC ranging between 0.41 and $1.65 \mu \mathrm{M}$ against M. tuberculosis, MDR-TB and XDR-TB strains. WGS identified mutations in the $c l p c 1$ gene in six spontaneous lassomycin-resistant $M$. tuberculosis colonies. This gene encodes the $\mathrm{ClpC1}$ subunit of the hexameric ATPase complex and this target was validated by measuring ATP hydrolysis and ATP-dependent protein breakdown by ClpP1P2. To understand the mechanism of action of lassomycin, a molecular docking approach was performed, focussing on the mutated sites revealed by WGS [54]. This study also highlighted the requirement to sequence multiple spontaneous mutants in order to demonstrate consistent genetic alterations in a putative target as against a background of genetic changes that would be unlinked to the resistance mechanism. Such a strategy could also reveal mechanisms based on multiple components acting synergistically. Thus, the increasing the number of mutant sequences would increase the likelihood of any inferred resistance mechanism being correct. Thus, six mutants were sequenced to suggest the lassomycin target but the actual number sequenced in other projects could be limited by financial resources.

\section{Proteomic approaches}

In situ identification of protein-molecule interactions is another valuable tool for the discovery of targets in a native cellular environment. These are based on advances in organic chemistry that have permitted the detection of target and off-target interactions by the modification of key 'activatable' groups of active molecules [55,56]. Chemical proteomics is based on the manipulation of a natural product of interest with a tag, normally a terminal alkyne, azide or cyclopropene, which will react via bio-orthogonal 'click' chemistry reaction with a fluorophore or affinity label. This reaction will occur after cells, previously incubated with the natural product, are lyzed. These terminal groups are small enough to not interfere negatively with the molecular binding between the target and the reactive site on the natural product. Biotin or a fluorophore can be directly attached to the natural product, instead of the tag, if permitted by their reactivity. The result of the reaction between the natural product and the fluorophore or affinity label can be analyzed by SDS-PAGE with in-gel fluorescence or LC-MS proteomics [57].

An affinity-based method was used to discover the target of agrimophol (Figure 3), a phloroglucinol from Agrimonia pilosa, which is a plant used in traditional Chinese medicine. Interestingly, this strategy was followed when no agrimophol-resistant mutants were obtained. Whole-cell based screens against $M$. tuberculosis found that agrimophol was able to inhibit $\mathrm{pH}$ homeostasis. A click-chemistry reaction was carried out to produce a biotinylated triazole and proteins were fractionated by SDS-PAGE. Following screening with the prebiotinylated probe a1b, the M. tuberculosis protein Rv3852; whose function is unclear, was suggested as the target of agrimophol; a conclusion that was further validated by protein mass fingerprinting [58]. A similar methodology was used to discover the target of cyclomarin A (Figure 3) produced by a marine bacterium (Streptomyces sp.) [59]. This is a cyclic peptide, identified from a whole-cell screening, and has MICs of 0.3 and $2.5 \mu \mathrm{M}$ against M. tuberculosis in culture broth and in human-derived macrophages, respectively [59]. In attempting the identification of the cyclomarin A target in M. tuberculosis, no spontaneous resistant mutants could be isolated. Therefore, a proteomics approach appeared to be an appropriate alternative method. Here, cyclomarin A was linked to sepharose beads and co-incubated with a M. bovis BCG cell lysate to enrich for the interacting target. The mixture was then separated using SDS-PAGE gels to reveal $\mathrm{ClpC1}$ was the major cyclomarin A interactor [59]. With the target now defined follow on studies revealed the co-crystal structure of the complex cyclomarin A-ClpC1. This will be indispensable to the design and synthesis of new antimycobacterials that target $\mathrm{ClpC} 1[60]$.

Another strategy that can be used to identify targets from natural products is drug affinity responsive target stability. It is based on the protective action against proteolysis conferred through target-protein interactions. Comparison of the degradation patterns in natural product interacting ('protected') samples versus 'unprotected' 


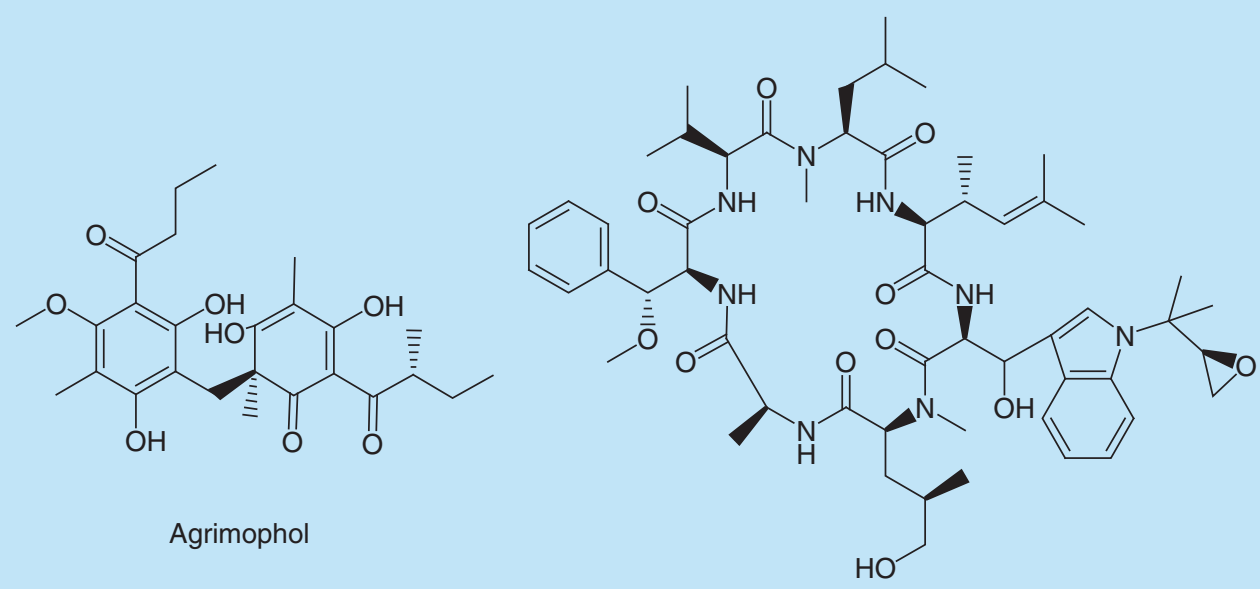

Cyclomarin A

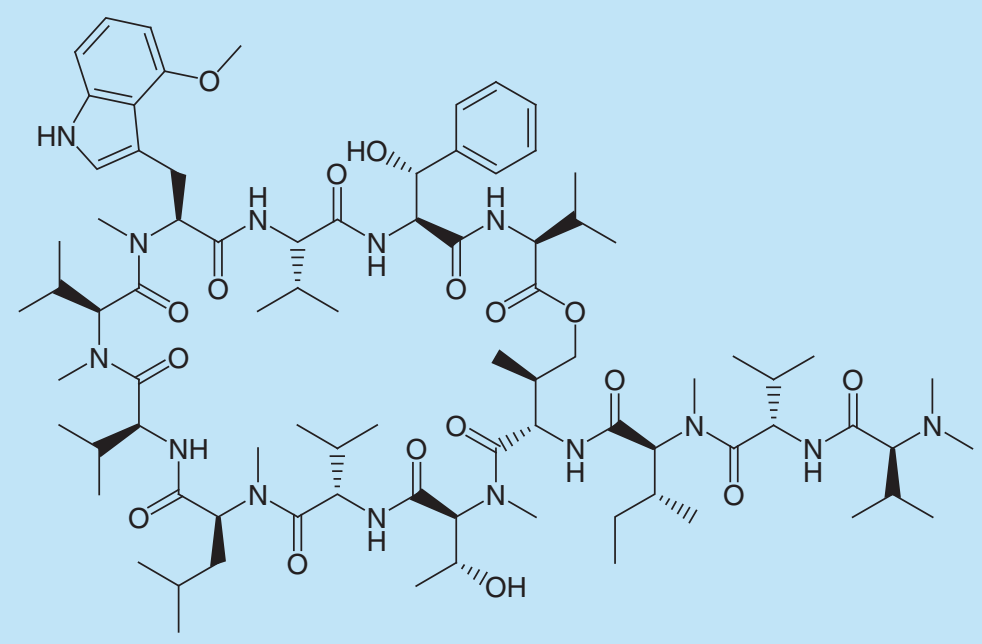

Ecumicin

Figure 3. Chemical structures of agrimophol, cyclomarin A and ecumicin.

controls can be used to suggest protein targets. This technique is known to be fast and avoids any costly structural modifications on the natural product as demanded in the techniques described previously.

Ecumicin (Figure 3), a macrocyclic tridecapeptide isolated from Nonomuraea sp. MJM5123, was discovered following high-throughput screening from different extracts of several actinomycetes [61]. It was highly active against M. tuberculosis, (MIC $0.26 \mu \mathrm{g} / \mathrm{ml}$ ) and against strains resistant to streptomycin, rifampicin and cycloserine [61] but its further development required definition of its mode of action within the bacteria. Initially, the genomic DNA of ecumicin-resistant strains of $M$. tuberculosis were sequenced and compared with the corresponding wild-type strain. As with agrimophol $\mathrm{ClpC1}$, was one of the targets found and was further validated using a proteomics-based test for drug affinity responsive target stability. Thus, ecumicin was able to protect $\mathrm{ClpC} 1$ from nonspecific proteolytic degradation in wild-type $M$. tuberculosis but not in the mutated form. Additional validation was made after LC-MS analysis of the excised band. In a logical, and important further step, Gao et al. co-crystallized ecumicin docked with $\mathrm{ClpC} 1$ which provided crucial information on the protein pocket required for interaction with the natural product and hence its molecular mechanisms of action. This information has facilitated the informed design of synthetic derivatives of ecumicin that could be more effective antibiotics [62]. 


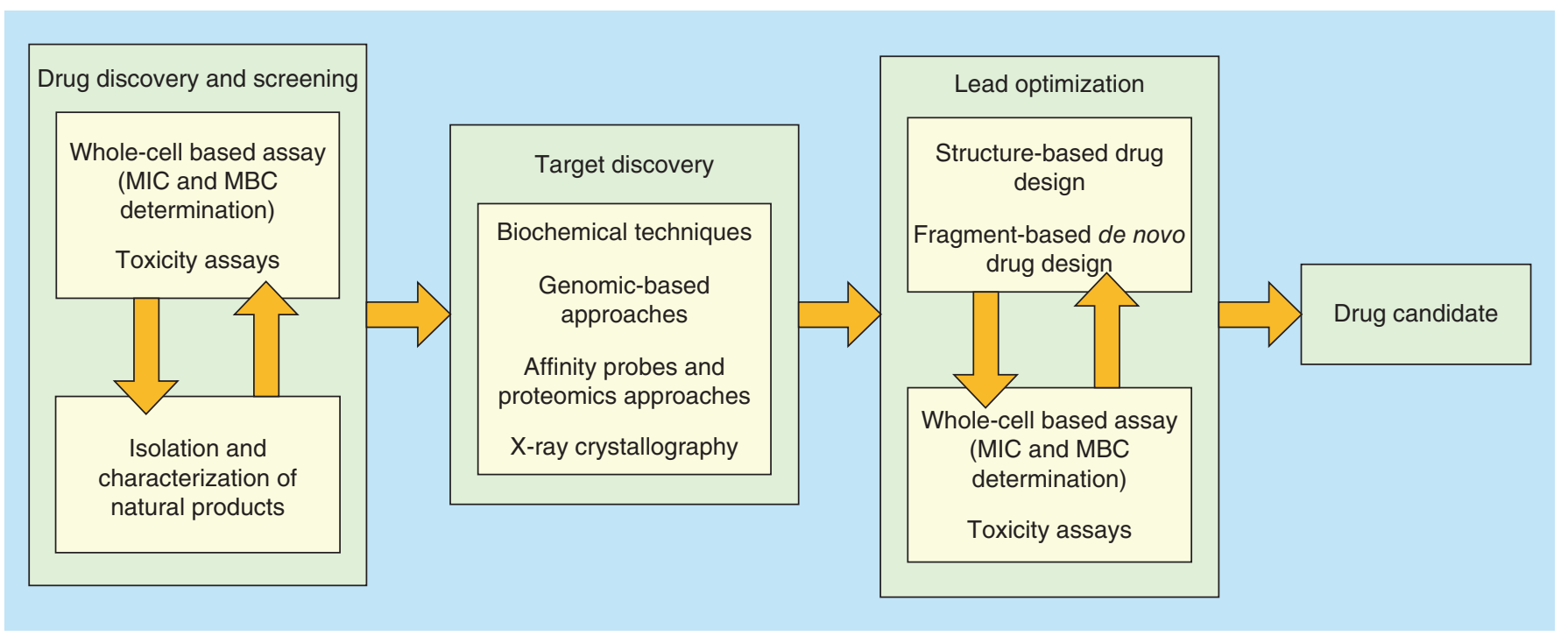

Figure 4. Strategies for antimycobacterial discovery pipeline from natural products. MBC: Minimal bactericidal concentration; MIC: Minimal inhibitory concentration.

\section{Computational chemistry strategies to inform \& optimize antimycobacterial natural products}

$\mathrm{X}$-ray crystallography can provide important insights on specific binding between drug and target through highresolution $3 \mathrm{D}$ structural data. For instance, $\mathrm{x}$-rays were used to confirm that $\mathrm{N}$-terminus of $\mathrm{ClpCq}$ bound lassomycin via positively charged guanidium groups. Docking studies showed that the N-terminal Gln17 was the major interacting residue through hydrogen binding. Moreover, lassomycin binding was reduced when $\mathrm{Gln} 17$ was mutated to Arg- or His [54].

The widespread use of $\mathrm{x}$-ray crystallography has led to a rapid increase in $3 \mathrm{D}$ protein structures suitable to use in in silico methods for hit identification and lead optimization. In addition to the computational chemistry methodologies that have been successfully applied on natural products to optimize drugs with antimycobacterial activity, other drug design techniques show considerable promise. Fragment-based de novo drug design, for instance, has enabled the redesigning of molecules, in a drug-target environment. This has led to the production of more active synthetic molecules based on the original scaffold and interactions [63]. However, this method has never been used in drug design of natural products against tuberculosis targets [64].

Different techniques in computational chemistry now also play important roles in optimization of a drug where previous research can be considered to only have provided a scaffold for further development. Once again natural products are often not taken forward to this important stage as no target is identified and validated and so will fail in the advancing the druggability of any identified bioactive. Thus, computerized approaches allow the visualization and analysis of 3D structures of the bioactive molecule and the target protein and their interactions. Based on these, it is possible to use virtual screening approaches to design derivatives, which allow better interaction between target and drug without altering its physical and chemical properties, assuring the drug is effective and not toxic and making synthesis more efficient. This was successfully applied with the generation and screening of new InhA inhibitors in silico [65].

Although, these approaches are powerful, they seldom can function ex nibilo in other words, design a drug based only on knowledge of the protein target pocket. Given this, natural product libraries play an important role in screening as they present a wide-ranging diversity of scaffolds complemented with a range of structural and functional features [66].

\section{Future perspective}

The examples given above have highlighted several approaches used to exploit natural products as new antimycobacterial drugs. Although, these present impressive progress, none has yet achieved the status of a commercially available drug. This reflects the relative ease that antimycobacterial natural products can be identified but is not matched by strategies where their targets are quickly defined. Without this, the value of a natural product as 
a drug lead cannot be assessed and, if suitable, further optimized. However, current research has also furnished approaches which can be rationalized into a robust discovery pipeline; often based on the use of the latest genomic and computational platforms (Figure 4). It should be noted that key steps in the pipeline, target discovery and lead optimization, encompass many approaches. Particularly with target discovery, adoption of multiple approaches at each step will undoubtedly increase the probability of successful definition of the targets, and not 'off-targets' for antimycobacterial activity. Therefore, the results of multiple strategies should be complementary and mutually confirmatory. This would also represent an efficient use of resources since the process of obtaining the natural products with their typically low yields, sometimes difficulty in confirming structures and complex/expensive synthetic routes, can impede progress. Given these points, research groups are urged to follow such a strategy to increase the probability of success; in other words, leading to drug candidates. This would allow the power of natural selection acting on natural products to be more effectively exploited in the search for new antimycobacterials.

\section{Executive summary}

Difficulties of target deconvolution

- High-throughput screenings of combinatorial libraries for target discovery have a poor hit rate.

- Natural products yield scaffolds that can be used as tools toward novel targets discovery.

Target discovery from natural products

- Natural products based research programs normally do not follow a complete pipeline of target discovery and validation.

- Whole-cell based screenings combined with '-omics' technologies make possible the discovery of new targets from new drug leads.

- Use of '-omics' allows small amount of sample to discover of new targets.

- Target discovery allows the consequent rational optimization of compounds or total synthesis by de novo techniques.

Financial \& competing interests disclosure

R Baptista would like to acknowledge the Life Sciences Research Network Wales for the PhD Studentship. S Bhowmick is thankful to Aberystwyth University for its AberDoc PhD Scholarship. IBERS receives strategic funding from the Biotechnology and Biological Sciences Research Council (BBSRC), UK. RJ Nash is CEO of Phytoquest. The authors have no other relevant affiliations or financial involvement with any organization or entity with a financial interest in or financial conflict with the subject matter or materials discussed in the manuscript apart from those disclosed.

No writing assistance was utilized in the production of this manuscript.

\section{References}

Papers of special note have been highlighted as: $\bullet$ of interest; $\bullet \bullet$ of considerable interest

1. WHO. 2016 Global tuberculosis report. WHO Press, Geneva, Switzerland. www.who.int/tb/publications/global_report/gtbr2016_executive_summary.pdf

2. Gandhi NR, Nunn P, Dheda K et al. Multidrug-resistant and extensively drug-resistant tuberculosis: a threat to global control of tuberculosis. Lancet. 375(9728), 1830-1843 (2010).

3. Yew WW, Lange C, Leung CC. Treatment of tuberculosis: update 2010. Eur. Respir. J. 37(2), 441-462 (2011).

4. Dartois V. The path of anti-tuberculosis drugs: from blood to lesions to mycobacterial cells. Nat. Rev. Microbiol. 12(3), 159-167 (2014).

5. Dorman SE, Chaisson RE. From magic bullets back to the magic mountain: the rise of extensively drug-resistant tuberculosis. Nat. Med. 13(3), 295-298 (2007).

6. Zumla A, Raviglione M, Hafner R, von Reyn CF. Tuberculosis. N. Engl. J. Med. 368(8), 745-755 (2013).

7. Chae L, Kim T, Nilo-Poyanco R, Rhee SY. Genomic signatures of specialized metabolism in plants. Science 344(6183), 510-513 (2014).

8. Janin YL. Antituberculosis drugs: ten years of research. Bioorg. Med. Chem. 15(7), 2479-2513 (2007).

9. Kana BD, Karakousis PC, Parish T, Dick T. Future target-based drug discovery for tuberculosis? Tuberculosis 94(6), 551-556 (2014).

- Comprehensive review of identification and validation of drug targets.

10. Cole ST, Riccardi G. New tuberculosis drugs on the horizon. Curr. Opin. Microbiol. 14(5), 570-576 (2011).

11. Munoz-Elias EJ, McKinney JD. Mycobacterium tuberculosis isocitrate lyases 1 and 2 are jointly required for in vivo growth and virulence. Nat. Med. 11(6), 638-644 (2005). 
12. Koul A, Arnoult E, Lounis N, Guillemont J, Andries K. The challenge of new drug discovery for tuberculosis. Nature 469(7331), 483-490 (2011).

13. Lechartier B, Rybniker J, Zumla A, Cole ST. Tuberculosis drug discovery in the post- post-genomic era. EMBO Mol. Med. 6(2), 158-168 (2014).

- Valuable critical review of tuberculosis drug discovery pipeline.

14. Servick K. The drug push. Science 348(6237), 850 LP-853 (2015).

15. Demain AL, Sanchez S. Microbial drug discovery: 80 years of progress. J. Antibiot. (Tokyo)62(1), 5-16 (2009).

16. Verdine GL. The combinatorial chemistry of nature. Nature 384 (6604 Suppl.), 11-13 (1996).

17. Stone MJ, Williams DH. On the evolution of functional secondary metabolites (natural products). Mol. Microbiol. 6(1), 29-34 (1992).

18. Dashti Y, Grkovic T, Quinn RJ. Predicting natural product value, an exploration of anti-TB drug space. Nat. Prod. Rep. 31(8), 990-998 (2014).

19. Farha MA, Brown ED. Strategies for target identification of antimicrobial natural products. Nat. Prod. Rep. 33(5), 668-680 (2016).

20. Santhosh RS, Suriyanarayanan B. Plants: a source for new antimycobacterial drugs. Planta Med. 80(1), 9-21 (2014).

21. Schenone M, Dančík V, Wagner BK, Clemons P. Target identification and mechanism of action in chemical biology and drug discovery. Nat. Chem. Biol. 9(4), 232-240 (2013).

-. Key report of techniques allowing target identification.

22. Herrmann J, Rybniker J, Müller R. Novel and revisited approaches in antituberculosis drug discovery. Curr. Opin. Biotechnol. 94-101 (2017).

23. Dong M, Pfeiffer B, Altmann K-H. Recent developments in natural product-based drug discovery for tuberculosis drug discovery. Today 22(3), 585-591 (2017).

24. Cheuka PM, Mayoka G, Mutai P, Chibale K. The role of natural products in drug discovery and development against neglected tropical diseases. Molecules 22(1), pii:E58 (2017).

25. Marri PR, Bannantine JP, Golding GB. Comparative genomics of metabolic pathways in Mycobacterium species: gene duplication, gene decay and lateral gene transfer. FEMS Microbiol. Rev. 30(6), 906-925 (2006).

26. Anishetty S, Pulimi M, Pennathur G. Potential drug targets in Mycobacterium tuberculosis through metabolic pathway analysis. Comput. Biol. Chem. 29(5), 368-378 (2005).

27. Noto T, Miyakawa S, Oishi H, Endo H, Okazaki H. Thiolactomycin, a new antibiotic. III. In vitro antibacterial activity. J. Antibiot. (Tokyo) 35(4), 401-410 (1982).

28. Kim P, Zhang Y-M, Shenoy G et al. Structure-activity relationships at the 5-position of thiolactomycin: an intact (5R)-isoprene unit is required for activity against the condensing enzymes from Mycobacterium tuberculosis and Escherichia coli. J. Med. Chem. 49(1), 159-171 (2006).

29. Machutta CA, Bommineni GR, Luckner SR et al. Slow onset inhibition of bacterial beta-ketoacyl-acyl carrier protein synthases by thiolactomycin. J. Biol. Chem. 285(9), 6161-6169 (2010).

30. Hayashi T, Yamamoto O, Sasaki H, Okazaki H, Kawaguchi A. Inhibition of fatty acid synthesis by the antibiotic thiolactomycin. J. Antibiot. 37(11), 1456-1461 (1984).

31. Slayden RA, Lee RE, Armour JW et al. Antimycobacterial action of thiolactomycin: an inhibitor of fatty acid and mycolic acid synthesis. Antimicrob. Agents Chemother. 40(12), 2813-2819 (1996).

32. Kremer L, Douglas JD, Baulard AR et al. Thiolactomycin and related analogues as novel anti-mycobacterial agents targeting KasA and KasB condensing enzymes in Mycobacterium tuberculosis. J. Biol. Chem. 275(22), 16857-16864 (2000).

33. Douglas JD, Senior SJ, Morehouse C et al. Analogues of thiolactomycin: potential drugs with enhanced anti-mycobacterial activity. Microbiology (2002), 3101-3109 (2016).

34. Lu X, Chen Y, You Q. 3D-QSAR, molecular docking studies, and binding mode prediction of thiolactomycin analogs as $\mathrm{mtFabH}$ inhibitors. J. Enzyme Inhib. Med. Chem. 25, 240-249 (2010).

35. Muller D, Krick A, Kehraus S et al. Brunsvicamides A - C: sponge-related cyanobacterial peptides with mycobacterium tuberculosis protein tyrosine phosphatase inhibitory activity. J. Med Chem. 49(16), 4871-4878 (2006).

36. Chen L, Zhou B, Zhang $S$ et al. Identification and characterization of novel inhibitors of $\mathrm{mPTPB}$, an essential virulent phosphatase from mycobacterium Tuberculosis. ACS Med. Chem. Lett. 1(7), 355-359 (2010).

37. Chlipala GE, Mo S, Orjala J. Chemodiversity in freshwater and terrestrial cyanobacteria - a source for drug discovery. Curr. Drug Targets 12(11), 1654-1673 (2011).

38. Gentry EJ, Jampani HB, Keshavarz-Shokri A et al. Antitubercular natural products: berberine from the roots of commercial Hydrastis canadensis powder. Isolation of inactive 8- oxotetrahydrothalifendine, canadine, $\beta$-hydrastine, and two new quinic acid esters, hycandinic acid esters-1 and -2. J. Nat. Prod. 61(10), 1187-1193 (1998). 
39. Boberek JM, Stach J, Good L. Genetic evidence for inhibition of bacterial division protein FtsZ by berberine. PLoS ONE 5(10), 1-9 (2010).

40. Liu YX, Xiao CL, Wang YX et al. Synthesis, structure-activity relationship and in vitro anti-mycobacterial evaluation of 13-n-octylberberine derivatives. Eur. J. Med. Chem. 52, 151-158 (2012).

41. Wang Y, Fu H, Li Y, Jiang J, Song D. Synthesis and biological evaluation of 8-substituted berberine derivatives as novel anti-mycobacterial agents. Acta Pharm. Sin. B. 2(6), 581-587 (2012).

42. Ricke DO, Wang S, Cai R, Cohen D. Genomic approaches to drug discovery. Curr. Opin. Chem. Biol. 10(4), 303-308 (2006).

43. Jaspars M, Pasupathy V, Crews P. A tetracyclic diamine alkaloid, halicyclamine A, from the marine sponge Haliclona sp. J. Org. Chem. 59(12), 3253-3255 (1994).

44. Pruksakorn P, Arai M, Kotoku N et al. Trichoderins, novel aminolipopeptides from a marine sponge-derived Trichoderma sp., are active against dormant mycobacteria. Bioorganic Med. Chem. Lett. 20(12), 3658-3663 (2010).

45. Tasdemir D, Topaloglu B, Perozzo R et al. Marine natural products from the Turkish sponge Agelas oroides that inhibit the enoyl reductases from Plasmodium falciparum, Mycobacterium tuberculosis and Escherichia coli. Bioorganic Med. Chem. 15(21), 6834-6845 (2007).

46. Arai M, Sobou M, Vilchéze C et al. Halicyclamine A, a marine spongean alkaloid as a lead for anti-tuberculosis agent. Bioorg. Med. Chem. 16(14), 6732-6736 (2008).

47. Arai M, Liu L, Fujimoto T, Setiawan A, Kobayashi M. DedA protein relates to action-mechanism of halicyclamine A, a marine spongean macrocyclic alkaloid, as an anti-dormant mycobacterial substance. Mar. Drugs 9(6), 984-993 (2011).

48. Pruksakorn P, Arai M, Liu L, Moodley P, Jacobs WRJ, Kobayashi M. Action-mechanism of trichoderin A, an anti-dormant mycobacterial aminolipopeptide from marine sponge-derived Trichoderma sp. Biol. Pharm. Bull. 34(8), 1287-1290 (2011).

49. Kavianinia I, Kunalingam L, Harris PWR, Cook GM, Brimble MA. Total synthesis and stereochemical revision of the anti-tuberculosis peptaibol trichoderin A. Org. Lett. 18(15), 3878-3881 (2016).

50. Payne DJ, Gwynn MN, Holmes DJ, Pompliano DL. Drugs for bad bugs: confronting the challenges of antibacterial discovery. Nat. Rev. Drug Discov. 6(1), 29-40 (2007).

51. Ioerger TR, O'Malley T, Liao R et al. Identification of new drug targets and resistance mechanisms in Mycobacterium tuberculosis. PLoS ONE 8(9), 1-13 (2013).

52. Pule CM, Sampson SL, Warren RM et al. Efflux pump inhibitors: targeting mycobacterial efflux systems to enhance TB therapy. J. Antimicrob. Chemother. 71(1), 17-26 (2016).

53. Hartkoorn RC, Sala C, Neres J et al. Towards a new tuberculosis drug: pyridomycin - nature's isoniazid. EMBO Mol. Med. 4(10), 1032-1042 (2012).

54. Gavrish E, Sit CS, Cao S et al. Lassomycin, a ribosomally synthesized cyclic peptide, kills mycobacterium tuberculosis by targeting the ATP-dependent protease ClpC1P1P2. Chem. Biol. 21(4), 509-518 (2014).

55. Bantscheff M, Scholten A, Heck AJR. Revealing promiscuous drug-target interactions by chemical proteomics. Drug Discov. Today 14(21-22), 1021-1029 (2009).

56. Bunnage ME, Chekler ELP, Jones LH. Target validation using chemical probes. Nat. Chem. Biol. 9(4), 195-199 (2013).

57. Raida M. Drug target deconvolution by chemical proteomics. Curr. Opin. Chem. Biol. 15(4), 570-575 (2011).

58. Zhao N, Sun M, Burns-Huang K et al. Identification of Rv3852 as an agrimophol-binding protein in mycobacterium Tuberculosis. PLoS ONE 10(5) (2015).

59. Schmitt EK, Riwanto M, Sambandamurthy V et al. The natural product cyclomarin kills Mycobacterium tuberculosis by targeting the ClpC1 subunit of the caseinolytic protease. Angew. Chem. Int. Ed. Engl. 50(26), 5889-5891 (2011).

60. Vasudevan D, Rao SPS, Noble CG. Structural basis of mycobacterial inhibition by Cyclomarin A. J. Biol. Chem. 288(43), 30883-30891 (2013).

61. Gao W, Kim JY, Chen SN et al. Discovery and characterization of the tuberculosis drug lead ecumicin. Org. Lett. 16(23), 6044-6047 (2014).

62. Gao W, Kim JY, Anderson JR et al. The cyclic peptide ecumicin targeting CLpC1 is active against Mycobacterium tuberculosis in vivo. Antimicrob. Agents Chemother. 59(2), 880-889 (2015).

63. Rodrigues T, Reker D, Schneider P, Schneider G. Counting on natural products for drug design. Nat. Chem. 8, $531-541$ (2016).

64. Newman DJ, Cragg GM. Natural products as sources of new drugs from 1981 to 2014. J. Nat. Prod. 79(3), 629-661 (2016).

65. Kouassi AF, Kone M, Keita M et al. Computer-aided design of orally bioavailable pyrrolidine carboxamide inhibitors of enoyl-acyl carrier protein reductase of Mycobacterium tuberculosis with favorable pharmacokinetic profiles. Int. J. Mol. Sci. 16(12), 29744-29771 (2015).

66. Breinbauer R, Vetter IR, Waldmann H. From protein domains to drug candidates - natural products as guiding principles in the design and synthesis of compound libraries. Angew. Chem. Int. Ed. Engl. 41(16), 2878-2890 (2002). 